\title{
TOWARD AUTOMATED FUNCTIONAL MODELING: AN ASSOCIATION RULES APPROACH FOR MINING THE RELATIONSHIP BETWEEN PRODUCT COMPONENTS AND FUNCTION
}

\author{
Tensa, Melissa; Edmonds, Katherine; Ferrero, Vincenzo; Mikes, Alex; Soria Zurita, Nicolas; \\ Stone, Rob; DuPont, Bryony \\ Oregon State University
}

\begin{abstract}
The objective of this research is to support DfX considerations in the early phases of design. In order to do conduct DfX, designers need access to pertinent downstream knowledge that is keyed to early stage design activities and problem knowledge. Product functionality is one such "key" connection between early understanding of the design problem and component choices which dictate product performance and impact, and repositories of design knowledge are one way to archive such design knowledge. However, curation of design knowledge is often a time-consuming activity requiring expertise in product modeling. In this paper, we explore a method to automate the populating of design repositories to support the overall goal of having up-to-date repositories of product design knowledge. To do this, we mine information from an existing repository to better understand the relationships between the components, functions, and flows of products. The resulting knowledge can be applied to automate functional decompositions once a product's components have been entered and thus reliably provide that "key" between early design activities and the later, component dependent characteristics.
\end{abstract}

Keywords: Functional modelling, Design for X (DfX), Machine learning, Data-driven design, Product respository

Contact:

DuPont, Bryony

Oregon State University

United States of America

bryony.dupont@oregonstate.edu

Cite this article: Tensa, M., Edmonds, K., Ferrero, V., Mikes, A., Soria Zurita, N., Stone, R., DuPont, B. (2019)

'Toward Automated Functional Modeling: An Association Rules Approach for Mining the Relationship between Product Components and Function', in Proceedings of the 22nd International Conference on Engineering Design (ICED19), Delft, The Netherlands, 5-8 August 2019. DOI:10.1017/dsi.2019.177 


\section{INTRODUCTION}

The modern global theater of product design has become increasingly complex. Designers today are challenged with creating and innovating designs that require more than simple problem-solving. Traditionally, product design has focused on the principle of designing for an intentional end-function of a product. Today's designers have additional design considerations to contend with such as how to improve the sustainability of a product, increase efficiency in use, reduce assembly time, reduce the product physical profile. These areas of design are termed $D f x$ or Design for $X$. The increasing complexity of product design promotes a need to improve the availability of design knowledge beyond the cognitive memory of modern designers. Design repositories are one way to increase auxiliary design knowledge, this data can be used to reduce design time, increase design uniformity, and increase design fidelity. A foundation of engineering design is functional decomposition. We hypothesize that providing designers with a better understanding of product functionality from data mining existing products, we can enable designers to identify component and function correlations early in the design process. We suggest that by bridging the gap between component and function in an automated way, we can offer external knowledge to aid in design creativity, fidelity, and increase solution breadth. This relationship between component and function will provide the foundation that will help designers identify how component and function influence all $D f x$ objectives.

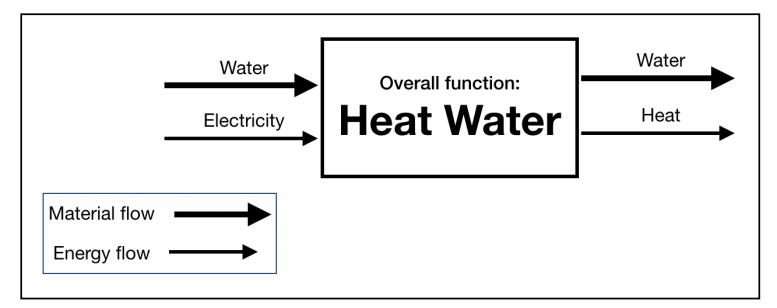

Figure 1. Black box electric tea kettle

Understanding functionality is an essential part of engineering design. Most product design efforts start with the need to design for an overarching function that solves a design problem. This overarching function can be considered a Black Box that is responsible for converting input flows into output flows, as shown for a traditional electric tea kettle in Figure 1. The designer is often able to intuit the input and output flows of a given black box design. For example, if tasked with creating an electric tea kettle, a designer will know the black box function is to heat water, and that this design will require inputs of electrical energy and water and outputs of thermal energy and heated water. The next step in the design process is to identify discrete system functions that help execute the main function. Identification of system functions is often completed through a functional decomposition. This decomposition is used to visualize the flows, internal secondary functions, and the intra-relations of discrete product functions, an example of which is shown in Figure 2. In addition to product functionality, the designer needs to determine the components and sub-assemblies that will make up the product. Component solutions are closely interrelated to function.

In this work, we explore how to increase and refine design knowledge by using association data mining on an existing Design Repository. This approach will enable us to understand the associations between product components and the probable functions they perform. To do this, we apply association rule learning using an Apriori algorithm to search repository data and determine the probabilities of relationships between components, functions, and flows within the Design Repository ${ }^{1}$, focusing on a subset of 12 Black and Decker products. These associations, once identified, can be used to inspire design of new products by allowing designer to access design a multitude of design solutions both typical and novel. This research also provides the basis for expanding design knowledge, as tied to our hypothesis, by first limiting the time required to add new products to the Design Repository. Second by enhancing auxiliary design knowledge, and providing component-based design suggestions based

1 The Design Repository is a database of design information compiled from over 130 products and housed at Oregon State University. A rudimentary web interface to access the information is available at: ftest.mime.oregonstate.edu/repo/browse 
on function. Furthermore, this work creates a prototype model for future work in automating functional modeling of user added products to the Design Repository.

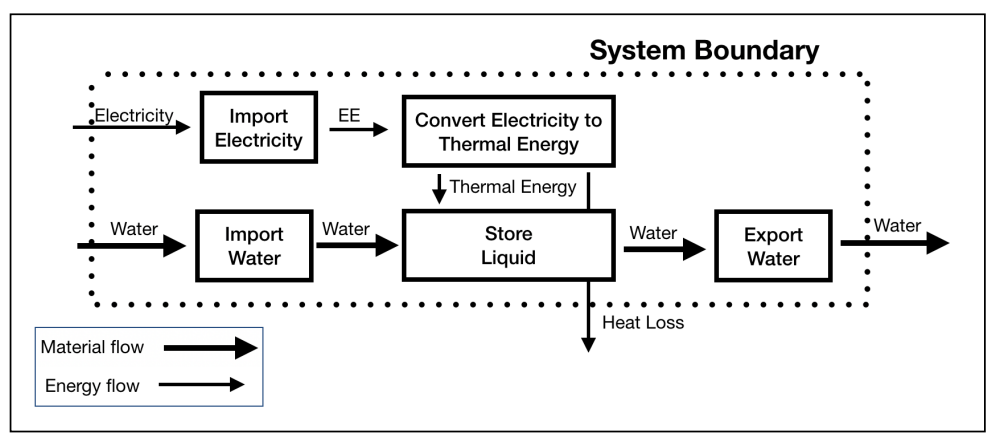

Figure 2. Simple functional model for an electric tea kettle

\section{LITERATURE REVIEW}

Design creativity is measured by the novelty, fluency and the number of ideas in a given design (Torrance 1962). Commonly used metrics to measure group idea generation are the quantity of non-redundant ideas and a quality rating (Mullen, Johnson, and Salas 1991). Shah et al. developed a set of metrics specifically for the evaluation of engineering idea generation techniques including quantity, quality, novelty, and variety of ideas (Shah, Smith, and Vargas-Hernandez 2003; Shah, Kulkarni, and Vargas-Hernandez 2000). They noted the fact that engineering design must meet a particular need and function thus requiring an expanded set of measures. Systematic design methodologies (Kevin N. Otto and Wood 2006; Pahl 2007; Ullman 2018; Cagan, Nussbaum, and Vogel 2002) provide a path for linking early design choices or decisions (e.g., customer needs gathering and interpretation, setting of engineering specifications) to an appropriately abstracted product function. The same methodologies and other stand-alone techniques offer a path to generate a set of component solutions from the derived functionality to define potential product variants.

\subsection{Internal \& external design search methods}

There are many different concept generation methods available to design engineers. These methods fall into two categories, internal and external searching methods. Well-known internal searching methods include brainstorming techniques, such as the 6-3-5 method and the function based morphological matrix introduced by Zwicky (K. Otto 2003; Zwicky 1969). A shortcoming of these and many other internal searching methods is the designer is limited to their own knowledge and there is a lack of external stimulation which may prompt the development of new avenues for creative thought.

An external search approach, referred to as catalog design, is based on a catalog of physical elements (components, assemblies, etc.) that can be browsed for solutions that match required performance specifications. A significant benefit of catalog design is the ability to utilize design knowledge that falls outside human memory (Roth 2002; Ward and Seering 1993). Designers of source inspiration from previous solutions in the concept generation phase (Eckert and Stacey 2000). One such example of a design catalog is a Design Repository.

Design repositories (S. Szykman et al., 2000) are currently still in their infancy, but their impact on data-driven design processes can be substantial. Currently the Design Repository at OSU is the largest known online Design Repository publicly available. Repositories store large datasets of products, providing a comprehensive collection of $D f X$ information which provides the fodder for predictive models to estimate downstream impacts earlier in the design process (Wisthoff et al. 2016). Previous researchers have used emerging technologies to enable a transition to the use of repositories in the design of products, through means such as driving concept generation through morphology (Wisthoff et al. 2016; Matt R. Bohm, Vucovich, and Robert B Stone 2008). Researchers have also explored the differences between design databases and repositories finding that repositories contain more comprehensive information and are dynamic. Repositories also provide the best means of gathering, recording, and storing artifact data (Matt R. Bohm, Robert B. Stone, and Simon Szykman 2005; Simon Szykman et al. 1999). 


\subsection{External design search methods: Oregon State University Design Repository}

In this study, the Design Repository hosted by the Design Engineering Lab at Oregon State University will be used, which contains information about 130 consumer based electro-mechanical products at various levels of abstraction (Matt R. Bohm, Haapala, et al. 2010; Matt R. Bohm, Vucovich, and Robert B Stone 2008; Matt R Bohm et al. 2008; Matt R. Bohm, Robert B. Stone, and Simon Szykman 2005). Product information is captured and separated into categories that allow innovative ways to approach design, with the goal of enabling designers to use data-driven methods to develop insight during the initial phase of concept generation. The underlying data schema captures a breadth of information, such as the color, system, function flow, manufacturing process, parameters, and other valuable information. The Design Repository stores product information as a PostgreSQL database.

Currently, the Design Repository contains over 5000 individual artifacts. Various iterations of the repository have refined the classification of the components and their functions to follow a standardized taxonomy (Matt R. Bohm, Haapala, et al. 2010; Matt R. Bohm, Vucovich, and Robert B Stone 2008; Matt R. Bohm, Robert B. Stone, and Simon Szykman 2005). In this work, we used the functional model with the functional basis and the component basis as the standardized design language (Matt R. Bohm and Robert B. Stone 2009; Robert B. Stone and Wood 2000). Artifacts are given both a common name and a specific component basis taxonomy name (Kurtoglu et al. 2009). For example, a common name of a component might be pin 1 and pin 2, but the component basis taxonomy would be pin for standardization of the dataset. The data from the repository can be extracted through queries that are written to return the information of interest. We queried the repository for an initial set of data which include the standardized component basis, the function it performs, and the flows in and out of the component for a set of Black and Decker power tools. This work is a preliminary analysis towards the verification of algorithms aimed at automatically generating a functional model based on an input of product components.

\subsection{Association rules \& Apriori algorithm}

Association rule mining is a form of data mining that searches information and determines the probabilities of relationships between variables (Borgelt 2012). In our analysis, we will utilize association rule mining, which finds finds non-obvious relationships between large datasets (Matt R. Bohm and Robert B. Stone 2009; Agrawal et al. 1993); most significantly, how one choice makes another choice more or less likely. In a supermarket example, an association rule may be that a purchaser buying hamburger buns is likely to also buy sliced cheese (Brin et al. 1997).

In product development, association rule mining (ARM) has been used to determine user preference of product functionality and product portfolio identification (Jiao and Zhang 2005; Agard and Kusiak* 2004). Association rule mining, in tandem with decision trees, can be used when developing convergent products that combine multiple user desired functions into a singular product (Lee, Song, and Park 2012). In terms of useful direct designer knowledge, ARM has been combined with user surveying and multi-objective genetic algorithms to generate soft rules for designs in specific product families. Rules such as: expected product shape, user preferences button shape (Fung et al. 2012). As shown, association rule mining can be used to identify important product functions, determine customer needs, and develop general design rules for new product development. These studies have provided a great understanding of the product design space, but are subject to drawbacks.

First, some of these studies are subject to imperfect or limited data, often relying on surveying techniques and/or online text mining (such as customer reviews). Though this type of data can capture the sentiment of the market, it may not prove useful information for developing generalized external design knowledge. The work presented in this paper, moves away from user need identification and uses vetted product system information from a Design Repository to disseminate useful external designer knowledge. Second, the literature presented focuses mainly on market identification. There is research gap between understanding the market, and the development of useful design knowledge. The aim of this work is to use ARM to offer novice designers the tools and information needed to design successfully in all product families. The case study presented in this paper is a stepping stone in providing: automated product functional modeling, suggest functions based on designer-supplied components, and provide suggestions on meeting designer-defined $D f x$ goals.

In our example, we will use association rule mining to identify the correlations between components and particular product functions; it is anticipated that each function will be associated with 
multiple components. The goal is to discover patterns in previous product designs, then use the patterns make rules determining the relationship between functions and flows for components. We use the Apriori algorithm, a common ARM algorithm, to find correlations within the Design Repository and establish statistically prevalent relationships for component-function-flow associations. Association rule mining requires large datasets to ensure validity, and we anticipate a set of 250 elements and a function set of 100 functions, resulting in a suitably large transaction space. The result of this task is a set of rules indicating the likelihood of components contributing to specific product functions. There are three main measurements that determine the associations, and these are derived through a probabilistic analysis of items and itemsets. In our research, an item is a component or a function, while an itemset is the combination of the component-function-flow that it performs. The first measure of association is called Support, which is the prevalence of an item within all of the itemsets. The second measure of association is called Confidence, which is the probability of two items appearing in the same item set. In the context of our work, Confidence is determined by the prevalence of combinations of components with functions and flows. The third measure of association is called Lift, which accounts for the popularity of the function and flow in the Confidence measurement of the combination with components. If a function and flow is popular, it could have a high Confidence, so Lift accounts for this so as not to conflate associations by simple prevalence.

\section{METHODOLOGY}

The focus of this work is to mine the Design Repository to better inform component-function-flow relationships, increase designer knowledge, and ultimately inform decisions made early in the design process. This goal is achieved by implementing association rule learning to gain insight into the current product data in the repository. Ultimately this work will allow for easier incorporation of new products into the Design Repository through automation. The methodology used in this paper is presented in Figure 3. We first query the repository database for the selected data; the components, functions, and flows from constituent products (for this particular study, twelve Black and Decker consumer products are selected). Then we apply association rules using an Apriori algorithm to the extracted set of data to determine the probabilities of associations between component basis terms (henceforth referred to as components), functions, and flows. The outputs of the algorithm are a list of components, the functions, and flows associated with that component, and the three measures of association: Support, Confidence, and Lift (previously defined in the literature review section). Finally, we apply these results to probabilistically determine the functions and flows for a given set of components for a new product.

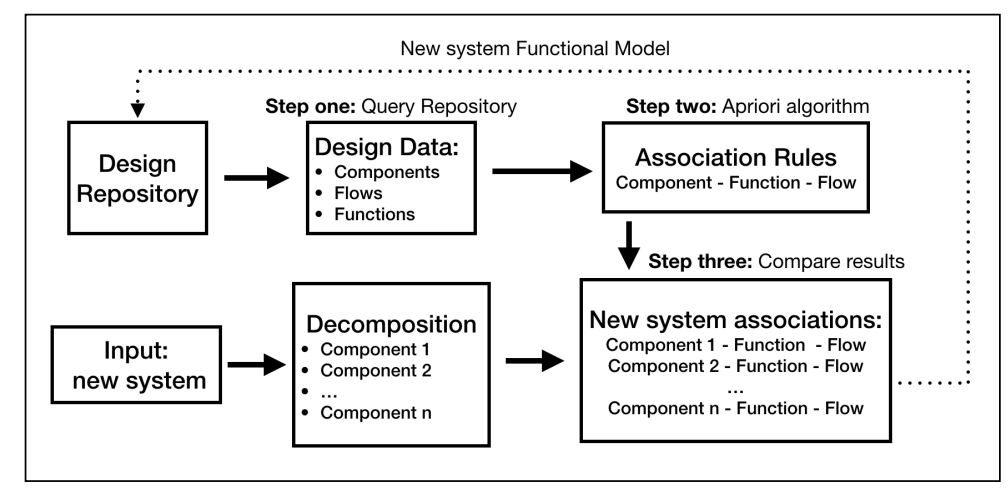

Figure 3. Methodology

The Apriori algorithm was selected as it is an efficient way to find association rules and allows verification of its effectiveness with a computationally simple example. The Apriori algorithm uses the measures of association to limit the number of itemsets that are explored based on a minimum threshold for Support and Confidence, thus decreasing computational complexity. The algorithm executes in Python using the library called PyFIM (Borgelt 2012). The Python Apriori algorithm requires an input of data as well as a declaration of minimum thresholds for the three measures of association: Support, Confidence, and Lift. The three measures of association: Support, Confidence, and Lift. Initially, these thresholds are set low in order to maximize the data returned to begin learning the relationships between components and functions. 


\subsection{Case study}

We apply the above methodology to a set of Black and Decker products extracted from the Design Repository. The Black and Decker subset of data was selected for analysis because the products have similar components and similar functionality, so we can find meaningful connections between component-function-flow associations through repetition. Additionally, we expect potential problems, as well as meaningful correlations, can be easily identified with a smaller set of data before applying these techniques to larger and more complex data. The Black and Decker dataset has twelve different tool systems and 50 unique components with 824 total combinations of components, functions, and flows, 152 of which are unique. The products include: sander attachment, can opener, circular saw attachment, drill attachment, dustbuster, jigsaw attachment, mini router attachment, power pack, screwdriver, slice right, palm sander, jigsaw, and rice cooker. We test automatic generation of component-function-flow relationships by applying the results of the Apriori algorithm to a list of components from a product in the Design Repository that was not analyzed with the Black and Decker data set. The componentfunction-flow information already exists and can easily be verified against. The verification case study product was a Delta jigsaw because it is in a similar design family as the Black and Decker products from the data set. Each unique component from the Delta Jigsaw was compared to the components in the results from the Black and Decker set to determine the most likely the function and flow. The result are compared to the component, function, and flows of the Delta Jigsaw to determine the accuracy of the algorithm. In summary, the steps to get these results are:

- Step One: Query the repository for component, function and flow combinations within a group of products.

- Step Two: Find most likely functions and flows for a component from association rules between the items (Apriori algorithm).

- Step Three: Compare results of the mined Black and Decker product component associations to the known function-component relationships and flows from the verification case study (the Delta jigsaw).

\section{RESULTS AND DISCUSSION}

\subsection{Black and Decker dataset}

The Black and Decker dataset was chosen for the similarity between product functions. There were a total of 866 combinations of components, functions, and flows with 283 unique combinations. The product similarity is indicated by the large number of total combinations, but smaller number of unique combinations. The small dataset provides more consistency in the results for this initial exploration in better understanding the steps toward automation. The Apriori algorithm found 67 associations between component, function, and flow to have 100\% Confidence, 49 associations between the values of $100 \%$ and $50 \%$, and 450 associations below the value of $50 \%$. The 11 associations with $100 \%$ Confidence have only one function and flow linked to them. For example, it was found that the component, bracket, will always be associated with the function secure and the flow solid. These high Confidence values create opportunities to easily develop rules that could be applied for automating functional modeling. Low Confidence values are the result of either a low Support value for a particular combination or the inclusion of many combinations in the dataset. Because most of the combinations are not unique, the Confidence measurement is not always the most indicative of a positive correlation between components, functions, and flows. When dividing each occurrence by the total number of combinations, Confidence for a few occurrences will become very small with a large dataset. This confirms the importance of using the Lift measurement, which accounts for the frequency of functions and flows when determining how meaningful a relationship is.

The two highest Lift values are 86,600, for the combinations of cushion - decrement rotational and diode - guide electrical. These both have $100 \%$ Confidence and very low support values, which means that these combinations only occurred once in our dataset. Extremely high values for Lift can also be an indication of a small dataset. Table 1 shows an example of our results for the component reservoir, all of the combinations have the same Confidence value, but the high Lift values are for function flow combinations that are more likely to occur in the dataset, such as store liquid and import liquid. The 
Confidence value is low for reservoir because it appears in the dataset in multiple combinations but with low prevalence for each.

Table 1. Reservoir values for lift and confidence

\begin{tabular}{c|c|c|c}
\hline Component & Function & Confidence & Lift \\
\hline \hline reservoir & store liquid & 0.1 & 82.4 \\
\hline reservoir & export mixture & 0.1 & 82.4 \\
\hline reservoir & import liquid & 0.1 & 82.4 \\
\hline reservoir & mix liquid & 0.1 & 82.4 \\
\hline reservoir & mix solid & 0.1 & 82.4 \\
\hline reservoir & store solid & 0.1 & 41.2 \\
\hline reservoir & export thermal & 0.1 & 20.6 \\
\hline reservoir & import solid & 0.1 & 3.582 \\
\hline
\end{tabular}

\subsection{Verification case study: Delta Jigsaw}

For this analysis, we featured five unique components that existed in the training dataset (Black and Decker) and in the verification case (the Delta jigsaw). Because the actual functions and flows of the jigsaw are known, the function and flows that the apriori algorithm outputs can be verified by direct comparison of results. Table 2 compares a sample of the results from the Black and Decker products and the Delta Jigsaw. The goal is to see how accurate the data is when compared to a product that was not included in the original learning dataset.

Table 2. Sample of verification dataset

\begin{tabular}{c|ccccc}
\hline \hline Head & Body & Confidence (\%) & Support (\%) & Lift (\%) & Jigsaw \\
\hline \hline Blade & export mechanical & 16.67 & 0.35 & 1443.33 & \\
& separate solid & 16.67 & 0.35 & 1202.78 & $\times$ \\
& export solid & 16.67 & 0.35 & 759.65 & $\times$ \\
& import solid & 16.67 & 0.35 & 601.39 & $\times$ \\
& transfer mechanical & 16.67 & 0.35 & 313.77 & $\times$ \\
& guide mechanical & 5.56 & 0.12 & 481.11 & \\
& secure solid & 5.56 & 0.12 & 54.67 & \\
& guide solid & 5.56 & 0.12 & 45.82 & \\
\hline Cam & change mechanical & 66.67 & 0.23 & 2510.14 & $\times$ \\
& transfer mechanical & 33.33 & 0.12 & 627.54 & $\times$ \\
\hline Screw & transfer electrical & 66.13 & 4.73 & 938.82 & $\times$ \\
& secure solid & 17.74 & 1.27 & 174.60 & \\
& position solid & 12.90 & 0.92 & 87.99 & \\
& guide solid & 3.23 & 0.23 & 26.61 & \\
& couple solid & 93.85 & 7.04 & 913.15 & $\times$ \\
& position solid & 6.15 & 0.46 & 41.96 & \\
& position solid & 47.37 & 1.04 & 323.00 & \\
& guide solid & 21.05 & 0.46 & 173.63 & $\times$ \\
& store mechanical & 10.53 & 0.23 & 4557.89 & \\
& supply mechanical & 10.53 & 0.23 & 4557.89 & \\
& secure solid & 5.26 & 0.12 & 51.79 & \\
& couple solid & 5.26 & 0.12 & 51.21 & \\
\hline
\end{tabular}

On the left side of Table 2, the Black and Decker dataset is shown with the Apriori results. The Confidence value for all combinations of components, functions, and flows for the learning dataset are scaled relative to each other by the same factor as Support. However, the Lift values scale the Confidence by the frequency of a function and flow combination. The right side of Table 2, indicated whether or not the jigsaw has the function and flow combination.

The table is organized by component from high to low Confidence. When there is an identical Confidence, it is then organized from high to low Lift. This reflects the importance we put on the different parameters. As the jigsaw results indicate, the associations with higher confidence or lift are indicating the more likely associations. We chose a sample of components from the larger jigsaw dataset. The results show the diversity of combinations between the learning set and the verification. For example, cam, as well as cap and washer, which was not shown, all had identical function and flows in the learning and verification set. The components blade and screw returned approximately twice as many 
associations for the Black and Decker set than then verification set. The component screw has a very high Confidence for couple solid (93.85) meaning it is significantly more likely to happen then position solid due to the higher Lifts and Supports. Lastly, the component spring only matched one function flow from the jigsaw, guide solid. The electric wire was the only component to have a function and flow that was not in the learning set. It has couple solid instead of secure, position, or guide solid. These are all very similar terminology, so there could be issues with standardized language. While there has been significant previous work to attempt to standardize language for functional basis and component basis taxonomy, no functional model is going to be created or replicated with $100 \%$ accuracy, so we see differences in the functional models. The Design Repository is built by different researchers, and their errors and subjectivity contribute to the variation in data in the repository, which is extracted into the results of automated analysis. The learning set contains products with different functionality than the jigsaw, this difference in functionality accounts for excess function and flow results in the learning set. However, these distinct results are important because they can provide the designer with novel solutions that they may not otherwise consider. The verification process using the Delta jigsaw has provided the first step towards automating functional modelling. However more data and analysis will be needed to be able to identify how many and which function and flows to choose from each component.

\section{CONCLUSIONS AND FUTURE WORK}

In this research, we used the Black and Decker dataset from the Design Repository to determine associations between component, function, and flow. The Apriori algorithm was implemented in Python to learn the association rules, which were compared to the Delta jigsaw, another product from the Design Repository. We found some similarities between the associations between the results from the Apriori algorithm and the Delta jigsaw, but our results were restricted by the limited size and scope of the data set used for this initial exploration. In future research, we will continue to query the Design Repository to expand our analysis to the entirety of the product information available, which will increase the accuracy of the association rule learning and assist in the eventual implementation of automatic functional modeling. As seen in our data, the higher the Confidence and lift values the more likely the combinations will appear in our verification data. Increasing our learning data set will help improve the validity of the association rules values, which will improve our confidence to assign function and flows to a particular component. This methodology could benefit other designers as it begins to bring to light to different potential concepts.

With improved component-function-flow association values, we can begin to develop grammar rules for applying the results of the associations that appear in our data. For example, in our Black and Decker dataset we found the component solder was always associated with the function-flow couple solid. These singular component, function and flow associations can be turned into grammar rules and coupled with additional data mining techniques to determine the order of function and flow in a functional model. A fairly straight forward example would be that a flow must always be imported before it is exported. While we applied this methodology specifically to a Design Repository, this methodology could be used on any product dataset to determine associations. Confidence and Lift values could be used to determine the most likely component and function and flow associations in the dataset used.

We plan to apply this methodology to the the entirety of the repository data in order to develop grammar rules and apply data mining to create a process of automated functional modeling. The results of association rule learning are the functional representation of a component. Given a component, we found the most likely functions and flows. Once these functional representations of each component are determined, they can be combined using grammar rules into the functional model. Automated functional modeling will allow for increased standardization in functional modeling and improved consistency between user created functional models. Furthermore, automation will increase the accuracy of generated function models, increase data fidelity, decrease decomposition time, and increase function data used to precipitate future research. This will also allow the repository adapt as trends in components change over time. One such avenue would be to create an algorithm that can automatically capture the functional model of an existing (or proposed) product then directly provide component and subassembly suggestions that relate to downstream impact of $D f X$ performance measures. This proposed research will increase the available auxiliary design knowledge which will improve innovation and creativity of designers, students, and other users of the repository. In this case, the algorithm will expand 
functional design solutions beyond the cognitive capabilities of the designer. Within the engineering education community, this approach could help with teaching the abstraction process that is critical to concept generation in systematic design approaches. Furthermore, we intend to verify automated supplied design knowledge through user feedback.

In the current state of the Design Repository, no information regarding the final user, the user interactions with the product, failure modes caused by the user, and humans error exists. Future work will include the development of new categories and relationships that include the user, user interactions, human error, and their relationships with the systems in the database expanding the work presented in the Function-Human Error Design Method (FHEDM) (Zurita et al. 2018). Such approach would enable safety, comfort, and performance objectives of the human-system interactions to become part of the up-front selection of design decision based on the human factors engineering assessment of user-system interactions.

\section{ACKNOWLEDGMENTS}

This material is based upon work supported by the National Science Foundation under Grant No. CMMI-1826469. Any opinions, findings, and conclusions or recommendations expressed in this material are those of the author(s) and do not necessarily reflect the views of the National Science Foundation.

\section{REFERENCES}

Agard, B. and Kusiak, A* (2004). "Data-mining-based methodology for the design of product families". In: International Journal of Production Research, Vol. 42 No. 15, pp. 2955-2969.

Agrawal, R. et al. (1993). "Mining association rules between sets of items in large databases". In: Proceedings of the 1993 ACM SIGMOD international conference on Management of data-SIGMOD '93. Vol. 22 No. 2. New York, New York, USA: ACM Press, pp. 207-216. ISBN: 0897915925. http://doi.org/10.1145/170035.170072. http://portal.acm.org/citation.cfm?doid=170035.170072.

Bohm, M.R. et al. (Sept. 2010). "Integrating Life Cycle Assessment Into the Conceptual Phase of Design Using a Design Repository". In: Journal of Mechanical Design, Vol. 132 No. 9, p. 091005. ISSN: 10500472. http://doi.org/10.1115/1.4002152. http://mechanicaldesign.asmedigitalcollection.asme.org/article. aspx?articleid=1450130.

Bohm, M.R. and Stone, R.B. (Jan. 2009). "A Natural Language to Component Term Methodology: Towards a Form Based Concept Generation Tool". In: Volume 2: 29th Computers and Information in Engineering Conference, Parts A and B. ASME, pp. 1341-1350. ISBN: 978-0-7918-4899-9. http://doi.org/10.1115/DETC2009-86581. http://proceedings.asmedigitalcollection. asme.org/proceeding.aspx?articleid= 1649365 .

Bohm, M.R. Stone, R.B. and Szykman, S. (Dec. 2005). "Enhancing Virtual Product Representations for Advanced Design Repository Systems". In: Journal of Computing and Information Science in Engineering, Vol. 5 No. 4, p. 360. ISSN: 15309827. http://doi.org/10.1115/1.1884618.

http://computingengineering. asmedigitalcollection.asme.org/article.aspx?articleid=1400359.

Bohm, M.R., Vucovich, J.P. and Stone, R.B. (2008). "Using a Design Repository to Drive Concept Generation". In: Journal of Computing and Information Science in Engineering. http://doi.org/10.1115/1.2830844. http://www.asme.org/about-asme/terms-of-use.

Bohm, M.R. et al. (2008). "Introduction of a data schema to support a design repository". In: Computer-Aided Design, Vol. 40 No. 7, pp. 801-811.

Borgelt, C. (2012). "Frequent item set mining". In: Wiley Interdisciplinary Reviews: Data Mining and Knowledge Discovery, Vol. 2 No. 6, pp. 437-456.

Brin, S. et al. (1997). "Dynamic itemset counting and implication rules for market basket data". In: Acm Sigmod Record, Vol. 26 No. 2, pp. 255-264. http://doi.org/10.1.1.41.6476.

Cagan, J., Nussbaum, B. and Vogel, C.M. (2002). Creating Breakthrough Products: Innovation from Product Planning to Program Approval. Financial Times Prentice Hall books. Prentice Hall PTR.

Eckert, C. and Stacey, M. (2000). "Sources of inspiration: a language of design". In: Design studies, Vol. 21 No. 5, pp. 523-538.

Fung, K.Y. et al. (2012). "A multi-objective genetic algorithm approach to rule mining for affective product design". In: Expert Systems with Applications, Vol. 39 No. 8, pp. 7411-7419.

Jiao, J. and Zhang, Y. (2005). "Product portfolio identification based on association rule mining". In: Computer-Aided Design, Vol. 37 No. 2, pp. 149-172. 
Kurtoglu, T. et al. (Mar. 2009). "A Component Taxonomy as a Framework for Computational Design Synthesis”. In: Journal of Computing and Information Science in Engineering, Vol. 9 No. 1, p. 011007. ISSN: 15309827. http://doi.org/10.1115/1.3086032.

http://computingengineering.asmedigitalcollection.asme.org/article.aspx?articleid=1401446.

Lee, C., Song, B. and Park, Y. (2012). "Design of convergent product concepts based on functionality: An association rule mining and decision tree approach". In: Expert Systems with Applications, Vol. 39 No. 10, pp. 9535-9542.

Mullen, B., Johnson, C. and Salas, E. (Mar. 1991). "Productivity Loss in Brainstorming Groups: A Meta-Analytic Integration". In: Basic and Applied Social Psychology, Vol. 12 No. 1, pp. 3-23. ISSN: 0197-3533. http://doi.org/10.1207/s15324834basp1201_1. https://www.tandfonline.com/doi/full/ 10.1207/s15324834basp1201\%7B\%5C_\%7D1.

Otto, K.N. and Wood, K.L. (2006). Product design: techniques in reverse engineering and new product development. Pearson Custom Pub.

Otto, K.N. (2003). Product design: techniques in reverse engineering and new product development.

Pahl, G. (2007). Engineering Design: a systematic approach. Springer.

Roth, K. (2002). "Design catalogues and their usage". In: Engineering Design Synthesis. London: Springer London, pp. 121-129. http://doi.org/10.1007/978-1-4471-3717-7_8. http://link.springer. com/10.1007/978-1-4471-3717-7\%7B\%5C_\%7D8.

Shah, J.J., Kulkarni, S.V. and Vargas-Hernandez, N. (Dec. 2000). "Evaluation of Idea Generation Methods for Conceptual Design: Effectiveness Metrics and Design of Experiments". In: Journal of Mechanical Design, Vol. 122 No. 4, p. 377. ISSN: 10500472. http://doi.org/10.1115/1.1315592.

$\mathrm{http}: / /$ mechanicaldesign.asmedigitalcollection. .asme.org/article. aspx? articleid=1446075.

Shah, J.J., Smith, S.M. and Vargas-Hernandez, N. (Mar. 2003). "Metrics for measuring ideation effectiveness". In: Design Studies, Vol. 24 No. 2, pp. 111-134. ISSN: 0142-694X.

http://doi.org/10.1016/S0142-694X(02)00034-0.

https://www.sciencedirect.com/science/article/pii/S0142694X02000340.

Stone, R.B. and Wood, K.L. (Dec. 2000). "Development of a Functional Basis for Design". In: Journal of Mechanical Design, Vol. 122 No. 4, p. 359. ISSN: 10500472. http://doi.org/10.1115/1.1289637. $\mathrm{http}: / /$ mechanicaldesign.asmedigitalcollection.asme.org/article.aspx?articleid=1446060.

Szykman, S. et al. (May 2000). "Design repositories: engineering design's new knowledge base". In: IEEE Intelligent Systems, Vol. 15 No. 3, pp. 48-55. ISSN: 1094-7167. http://doi.org/10.1109/5254.846285. http://ieeexplore.ieee.org/document/846285/.

Szykman, S. et al. (1999). "The NIST Design Repository Project". In: Advances in Soft Computing. London: Springer London, pp. 5-19. http://doi.org/10.1007/978-1-4471-0819-1_2. http://link.springer.com/10.1007/978-1-4471-0819-1\%7B\%5C_\%7D2.

Torrance, E.P. (1962). Guiding creative talent. Englewood Cliffs: Prentice-Hall, Inc. http://doi.org/10.1037/13134-000. http://content.apa.org/books/13134-000.

Ullman, David G. (2018). The mechanical design process. David G. Ullman.

Ward, A.C. and Seering, W.P. (Mar. 1993). "Quantitative Inference in a Mechanical Design 'Compiler"'. In: Journal of Mechanical Design, Vol. 115 No. 1, p. 29. ISSN: 10500472. http://doi.org/10.1115/1.2919320. http://mechanicaldesign.asmedigitalcollection.asme.org/article.aspx?articleid=1443468.

Wisthoff, A. et al. (Aug. 2016). "Quantifying the Impact of Sustainable Product Design Decisions in the Early Design Phase Through Machine Learning”. In: Volume 4: 21st Design for Manufacturing and the Life Cycle Conference; 10th International Conference on Micro- and Nanosystems. ASME, V004T05A043. ISBN: 978-0-7918-5014-5. http://doi.org/10.1115/DETC2016-59586. http://proceedings.asmedigitalcollection.asme.org/proceeding.aspx?doi=10.1115/DETC2016-59586.

Zurita, N.F.S. et al. (2018). "The Function-Human Error Design Method (FHEDM)". In: ASME 2018 International Design Engineering Technical Conferences and Computers and Information in Engineering Conference. American Society of Mechanical Engineers, V007T06A058-V007T06A058.

Zwicky, F. (1969). "Discovery, invention, research through the morphological approach". In: https://philpapers.org/rec/ZWIDIR. 